\title{
Spinal Intradural Hematoma and Permanent Paraparesis after a Lumboperitoneal Shunt Operation: An Unusual Complication
}

\author{
Recep Basaran ${ }^{1}$, Mustafa Efendioglu ${ }^{2}$, Fatih Han Bolukbasi ${ }^{3}$, Sahin Aslan ${ }^{2}$, Nejat Isik ${ }^{2}$, Tuncay Kaner ${ }^{2}$ \\ ${ }^{1}$ Department of Neurosurgery, Dr. Ismail Karakuyu Simav State Hospital, Kutahya, Turkey \\ ${ }^{2}$ Department of Neurosurgery, Istanbul Medeniyet University Goztepe Education and Research Hospital, Istanbul, Turkey \\ ${ }^{3}$ Department of Neurosurgery, Sorgun State Hospital, Yozgat, Turkey
}

Pseudotumor cerebri is a condition of increased intracranial pressure in the absence of clinical, laboratory or radiological pathology. Spinal intradural hematoma formation after lumboperitoneal shunt (LPS) implantation is very rare, but it can cause sudden and serious deterioration. In this report, we present a patient who developed an intradural hematoma following LPS operation. A 27-year-old male patient suffering from headaches and progressive vision loss was diagnosed with pseudotumor cerebri. He underwent LPS operation in January 2009. Four hours after the operation, he developed urinary and fecal incontinence with paraparesis (1/5). Lumbar magnetic resonance imaging identified an intradural hematoma at the level of $L 2-L 3$, and he was reoperated. The intradural hematoma was removed. Physical therapy was started because of paraparesis. Two months later, the patient's muscle strength had increased to $3 / 5$. Surgeons must remember that, LPS implantation can cause a spinal intradural hematoma in a small percentage of patients, with catastrophic results.

Keywords: Pseudotumor cerebri; Lumboperitoneal shunt; Intradural hematoma; Paraparesis; Cauda equina syndrome

\section{Introduction}

Pseudotumor cerebri (PTC) is a disease characterized by increased intracranial pressure in the absence of radiological intracranial pathology. PTC typically presents in middle-aged obese women. It causes increased intracranial pressure-related symptoms, such as headaches, pulsatile tinnitus, transient vision loss and diplopia [1].

The first-line treatment of PTC includes diuretic drugs, steroids and recurrent lumbar punctures [2]. Pseudotumor cerebri often deteriorates despite medical treatment and lumbar punctures and causes vision loss. Thus, it requires neurosurgical intervention. Ventriculoperitoneal shunts and lumbo-peritoneal shunts are among the surgical treatment options.

No complications have been reported in the literature after lumbo-peritoneal shunt implantation, except for headaches due to the lumbar puncture. Infection and shunt dysfunction are very rare consequences of the implantation. However, epidural, subdural, subarachnoid and intracerebral hemorrhages have been cited as rare complications of lumbar punctures [3]. Cerebrospinal fluid fistulas primarily occur in children [4]. The risk of spinal intradural hematoma development is prominent

Received Mar 28, 2013; Revised Aug 29, 2013; Accepted Sep 22, 2013

Corresponding author: Recep Basaran

Department of Neurosurgery, Dr. Ismail Karakuyu Simav State Hospital, Kutahya, Turkey

Tel: +90-506-659-72-21, Fax: +90-274-513-67-01, E-mail: drrecepbasaran@gmail.com 
in the presence of lumbar surgery of multiple levels, anatomical abnormalities, trauma during puncture, coagulation defects or the use of anticoagulant drugs $[5,6]$. Hematoma is occasionally observed after vertebral spine trauma [7] and even spontaneously [8]. Although spinal intradural hemorrhages can develop as a result of many causes, they are observed very rarely in this situation. However, when a spinal intradural hemorrhage does occur, it causes sudden and serious neurological deterioration [9].

In this report, we describe a patient who underwent lumboperitoneal shunt implantation due to pseudotumor cerebri and developed a spinal intradural hematoma in the early postoperative state. Although we performed early blood evacuation from the hematoma, the patient developed a permanent neurological deficit.

\section{Case Report}

A 27-year-old male patient presented at our hospital in September 2008. He was suffering from a sudden onset of headache that had awoken him from sleep and increased with eye movements. He also had vision loss in his left eye. A neurological examination identified only bilateral papilledema, and a visual field examination demonstrated bilateral (more prominent on the right side) peripheral narrowing. He had no known systemic disease and no drug use, except for amoxicillin one week prior to his presentation.
All tests performed to determine the symptom etiology were negative. His cranial magnetic resonance imaging (MRI) was within normal limits. Cranial MRI venography demonstrated that the left transverse sinus and sigmoid sinus were hypoplastic, and the right side was dominant (MRI venography) (Fig. 1). The patient did not benefit from medical treatment. He underwent daily lumbar punctures for one week. The opening cerebrospinal fluid (CSF) pressure was above $40 \mathrm{~cm} \mathrm{H}_{2} \mathrm{O}$. The patient's headaches improved, but his vision loss remained constant.

The patient was diagnosed with PTC. We took him into surgery and implanted a lumbo-peritoneal shunt in January 2009. The operation was carried out under general anesthesia in the left lateral decubitus position, with bilateral lower limbs in the bent position. The L2-3 intervertebral space was selected as the point of puncture, through which a $1-\mathrm{cm}$ opening was made with a sharp knife. A modified spinal puncture needle was inserted 5-7 $\mathrm{cm}$ along the midline. When an obvious breakthrough feeling was noted, this indicated that the needle was penetrating the spinal dura mater, at which point the needle core was withdrawn until the needle had been inserted through the subarachnoid cavity, with CSF flowing. Meanwhile, the shunt tube was inserted 7-9 $\mathrm{cm}$ into the subarachnoid cavity through the spinal puncture needle, and when CSF flowed through the shunt tube, the spinal puncture needle was pulled out. The shunt tube was tunneled to $2 \mathrm{~cm}$ lateral to the umbilicus on the lower right abdominal wall. This was penetrated with the puncture

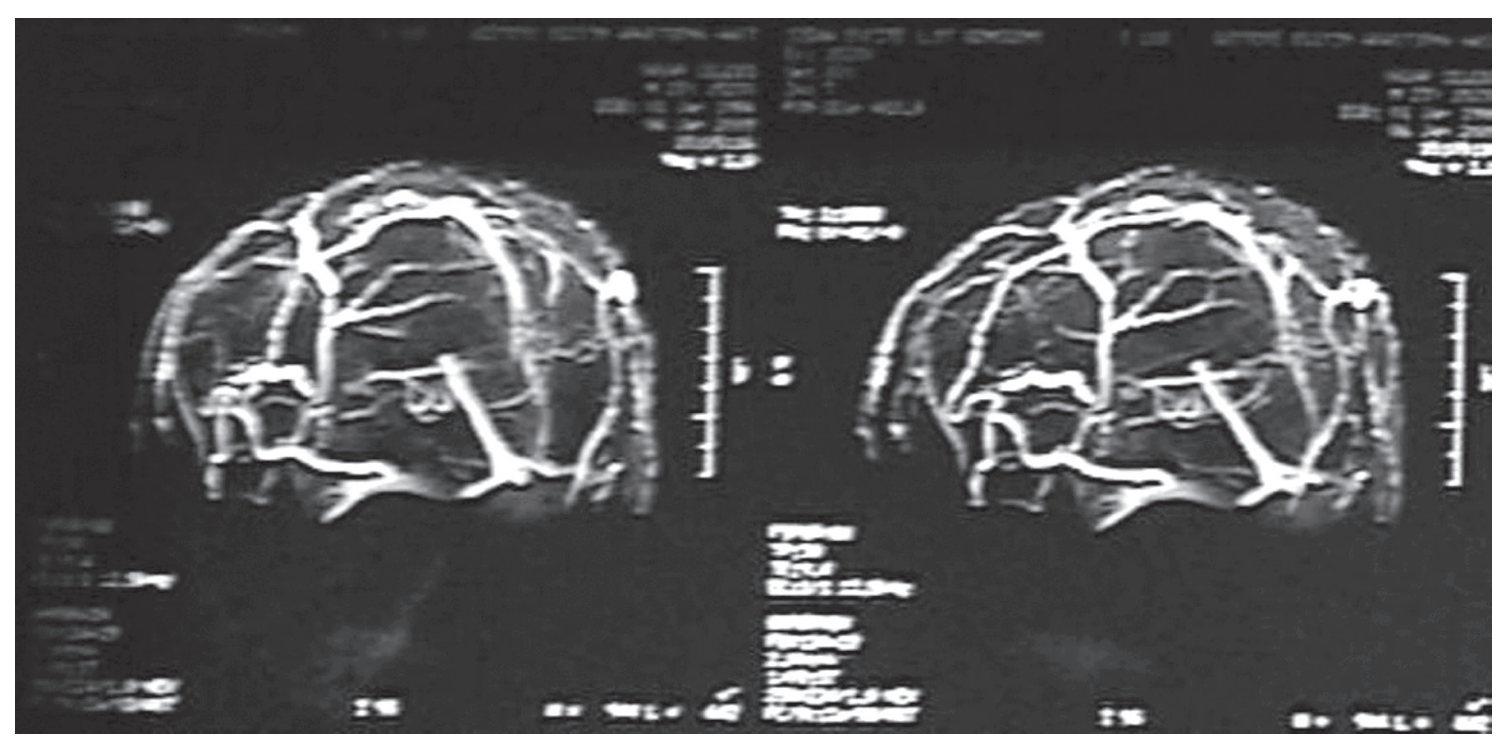

Fig. 1. Cerebral venograpy showing occlusion of the right transverse sinus. 
needle towards the lower and inward direction. When

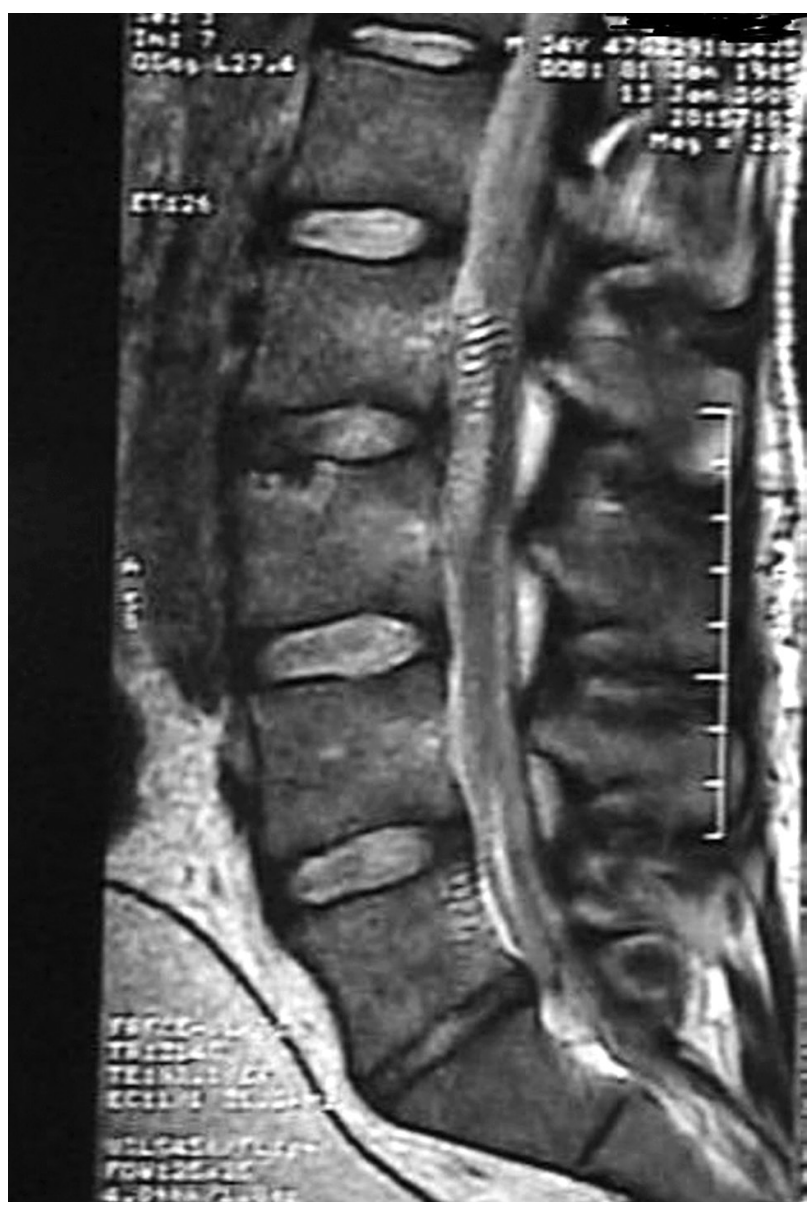

Fig. 2. Lumbar sagittal magnetic resonance imaging showing an intradural hematoma at the $\mathrm{L} 2-\mathrm{L} 3$ vertebral level. an obvious breakthrough feeling was noted, the needle core was withdrawn and the abdominal end of the shunt tube was inserted $20 \mathrm{~cm}$ via the puncture needle. Sutures were applied to fix the shunt valve and shunt tube in place, which aided in the avoidance of shifting or movement. Finally, the incision was sutured. Four hours after the operation, he developed urinary and fecal incontinence with paraparesis (1/5). Lumbar MRI identified an intradural hematoma at the level of L2-L3 (Figs. 2, 3), and the patient was taken back into surgery. Firstly, L2-L3 total laminectomy was performed. A dural defect was detected on the needle's entrance zone into the dura. Subsequently, the dura was opened. An arterial injury caused by the needle at the inner side of the dura was observed. The intradural hematoma was removed totally (Figs. 4, 5). During removal of his shunt, a CSF fistula occurred, and dural repair was performed. The family members of the patient refused a second shunt implantation. Physical therapy was started because of the lower extremity weakness. Two months after the operation, the patient's muscle strength had increased to $3 / 5$. A clean intermittent catheterization was performed for urinary incontinence. At the final neurological examination of the patient, he had light perception in his right eye, paraparesis (3/5) and urinary incontinence.

\section{Discussion}

Pseudotumor cerebri is a disease manifesting with headache and blurred vision. When left untreated, it causes

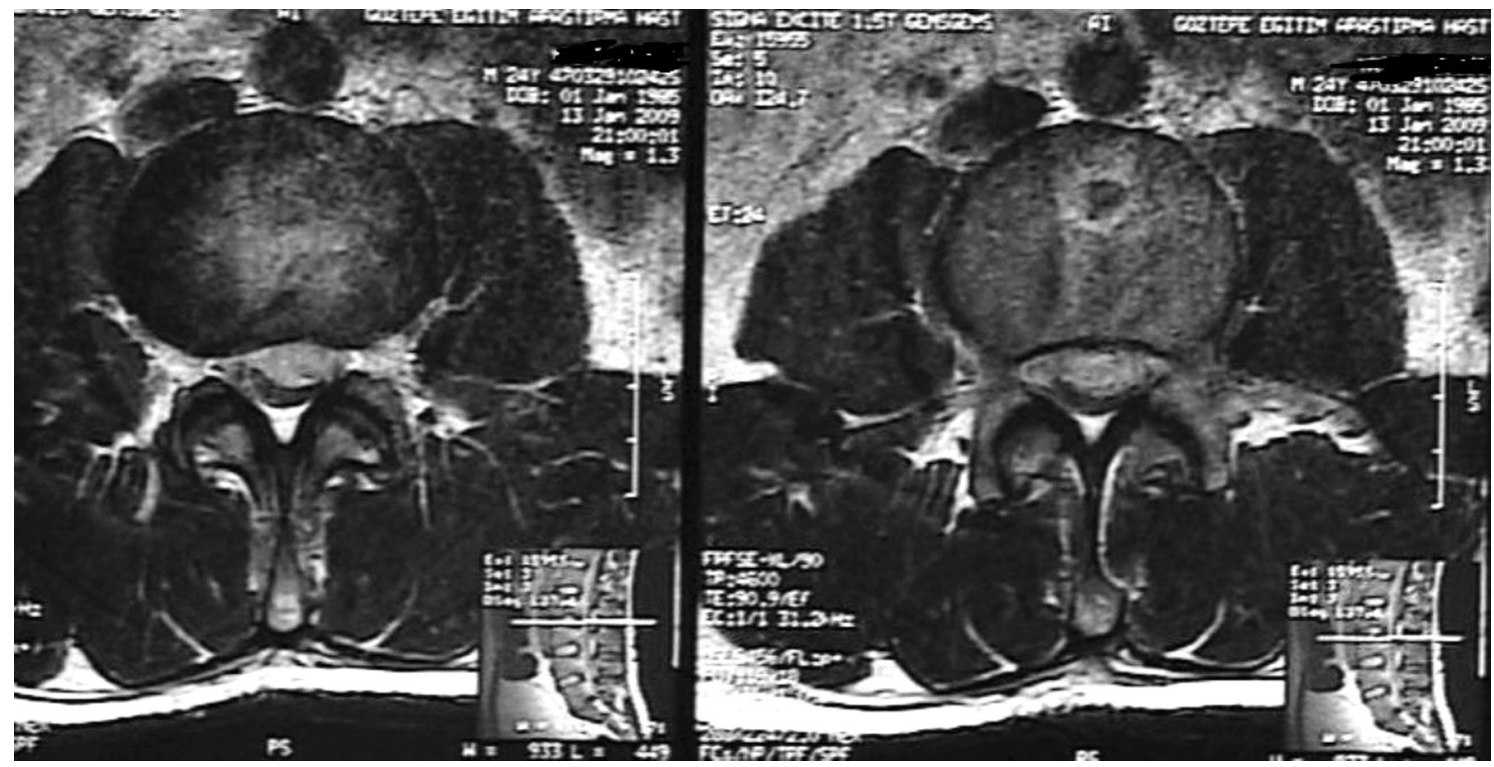

Fig. 3. Lumbar axial magnetic resonance imaging showing an intradural hematoma at the L2-L3 intervertebral disc level. 


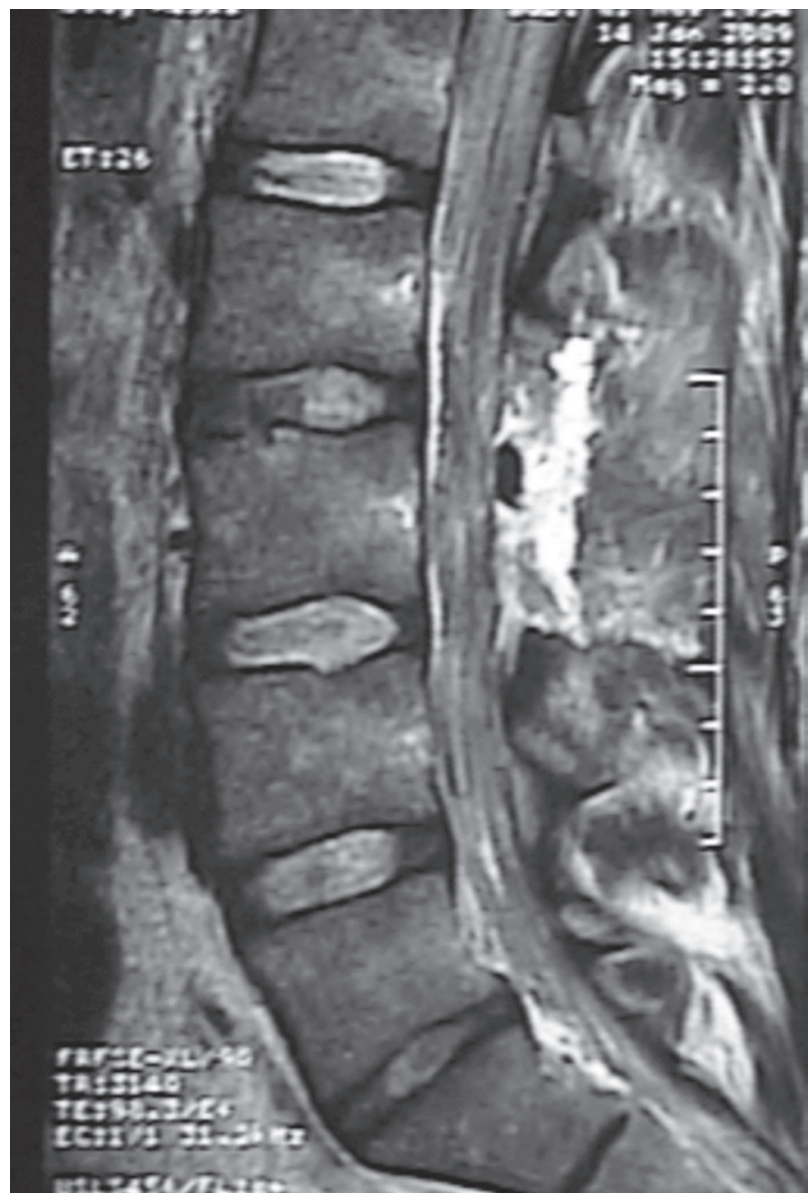

Fig. 4. Postoperative lumbar sagittal magnetic resonance imaging at the $\mathrm{L} 2-\mathrm{L} 3$ vertebral level. progressive vision loss and, finally, total amaurosis. Its pathophysiology is not fully understood. Etiologically, occlusion in the major venous sinuses, growth hormone treatment, hypervitaminosis $\mathrm{A}$, hypovitaminosis $\mathrm{A}$, oral contraceptive use and a sudden decrease in corticosteroid levels are held responsible for the disease. Acetazolamide, lumbo-peritoneal shunt implantation and optic nerve sheath decompressionare performed for treatment [10].

The implantation of a lumbo-peritoneal shunt is performed by guiding the shunt to the subdural space through a lumbar puncture. The other edge of the shunt is placed in the peritoneum. A lumbar puncture is often used for CSF examination and spinal anesthesia. Despite its commonness, there are no reported cases of intraspinal hemorrhage with permanent neurological deterioration due to a lumbar puncture in the absence of coagulopathy. Kaner et al. [11] experienced a hardly controlled hemorrhage during spinal surgery. However, in their case, the patient had a congenital fibrinogen deficiency. Intraspinal hematoma can occur in patients with coagulopathy [5], in anticoagulant or thrombolytic drug users $[5,6]$ and in children with leukemia [12]. In some cases, even spontaneous intradural bleeding is noted $[5,6]$.

Spinal intradural or epidural hemorrhages are wellknown complications of spinal surgery $[5,13]$. However, the prevalence of hemorrhages resulting in permanent

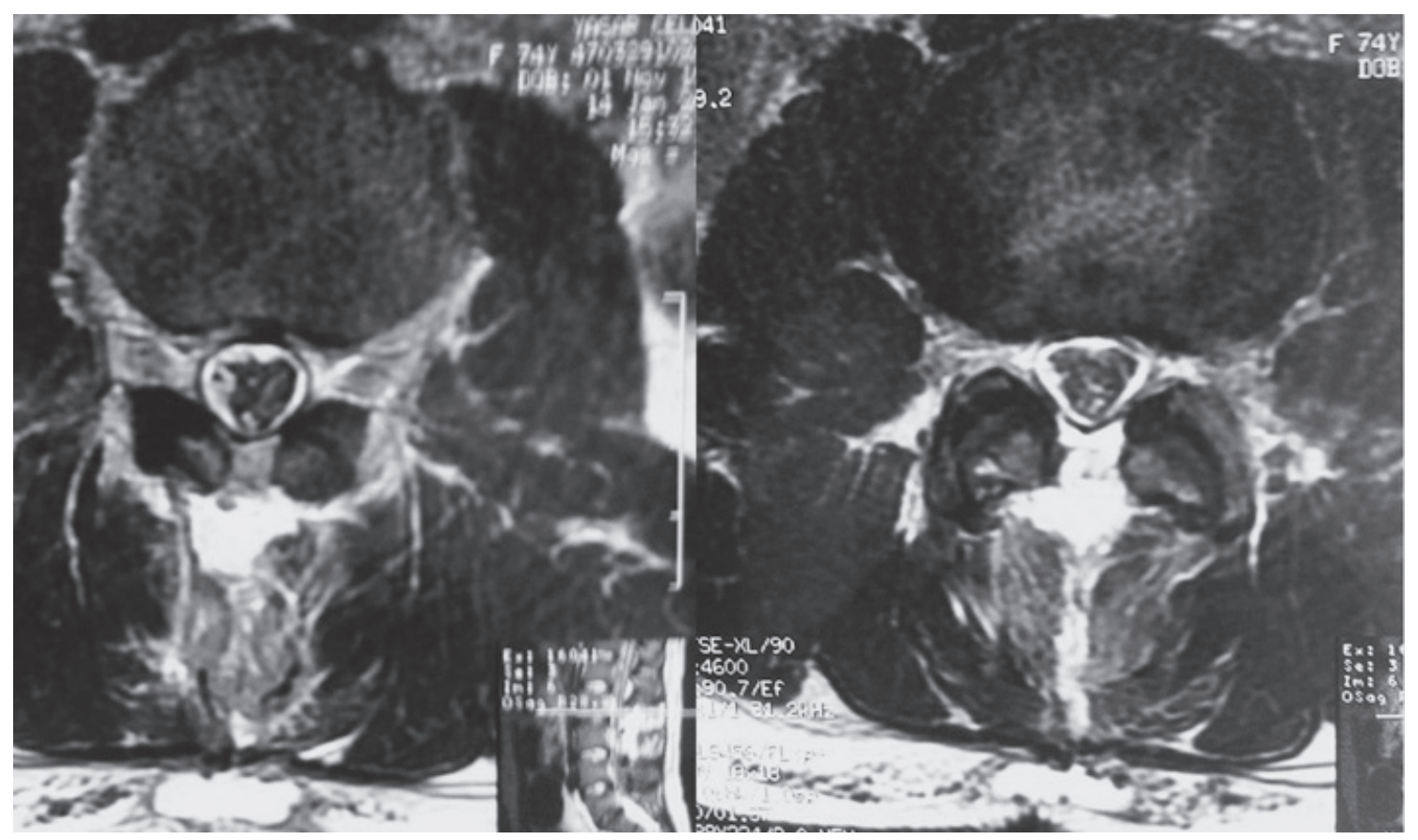

Fig. 5. Postoperative lumbar axial magnetic resonance imaging at the $L 2-L 3$ intervertebral disc level. 
neurological deficits is very low. According to Lawton et al. [14], its prevalence is only $0.1 \%$. In such situations, we expect a postoperative late-onset neurological deficit and its deterioration or the sudden development of cauda equina syndrome [13]. Our patient developed urinary and fecal incontinence four hours after the operation, accompanied by progressive paraparesis. This type of hemorrhage is a serious complication, regardless of its etiology. Thus, quick diagnosis and decompression are crucial. According to Vandermeulen et al. [15], spinal intradural hemorrhages have good outcomes when decompression is performed within the first 8 hours. Although our patient underwent the procedure within two hours of the development of the neurological deficit, he failed to show amelioration of the urinary and fecal incontinence and paraparesis. The occurrence of a hemorrhage in our patient was due to bleeding from the small arteries of the ligamentum flavum or from the dural or spinal cordsupporting arteries. The perioperative bleeding in our patient was caused by lumbar puncture needles damaging the supporting arteries located intradurally.

Lumbar punctures are very commonly practiced by physicians. During a lumbar puncture, intradural bleeding due to a damaged dural artery is a rare and unfortunate situation for the physician, but it can be even more unfortunate for the patient. The importance of patient follow up, even for the smallest invasive procedures, was revealed by our case. Surgeons should remember that a lumbo-peritoneal shunt implantation used to treat diseases such as pseudotumor cerebri, which has catastrophic results when left untreated even though it is very rare, may cause a spinal intradural hematoma and result in high morbidity.

\section{Conflict of Interest}

No potential conflict of interest relevant to this article was reported.

\section{References}

1. Fernando RR, Mehta NN, Fairweather MG. Pseudotumor cerebri and ciprofloxacin: a case report. J Med Case Rep 2011;5:104.

2. Kumar N, Bierbrauer KS, Grewal H. Microlaparoscopic-assisted lumboperitoneal shunt in the lateral position for pseudotumor cerebri in a morbidly obese adolescent. JSLS 2006;10:267-9.

3. Koch BL, Moosbrugger EA, Egelhoff JC. Symptomatic spinal epidural collections after lumbar puncture in children. AJNR Am J Neuroradiol 2007;28:1811-6.

4. Kiechl-Kohlendorfer U, Unsinn KM, Schlenck B, Trawoger R, Gassner I. Cerebrospinal fluid leakage after lumbar puncture in neonates: incidence and sonographic appearance. AJR Am J Roentgenol 2003; 181:231-4

5. Kou J, Fischgrund J, Biddinger A, Herkowitz H. Risk factors for spinal epidural hematoma after spinal surgery. Spine (Phila Pa 1976) 2002;27:1670-3.

6. Gurkanlar D, Acikbas C, Cengiz GK, Tuncer R. Lumbar epidural hematoma following lumbar puncture: the role of high dose LMWH and late surgery. A case report. Neurocirugia (Astur) 2007;18:52-5.

7. Stewart DH Jr, Watkins ES. Spinal cord compression by chronic subdural hematoma. Case report. J Neurosurg 1969;31:80-2.

8. Cloward RB, Yuhl ET. Spontaneous intraspinal hemorrhage and paraplegia complicating dicumarol therapy. Neurology 1955;5:600-2.

9. Lerner SM, Gutterman P, Jenkins F. Epidural hematoma and paraplegia after numerous lumbar punctures. Anesthesiology 1973;39:550-1.

10. Friedman DI, Jacobson DM. Diagnostic criteria for idiopathic intracranial hypertension. Neurology 2002;59:1492-5.

11. Kaner T, Ozer AF, Sasani M, Oktenoglu T, Alemdag S. The erioperative importance of congenital fibrinogen deficiency in spinal surgery procedure: a case report. Eur J Gen Med 2010;7:426-8.

12. Wolcott GJ, Grunnet ML, Lahey ME. Spinal subdural hematoma in a leukemic child. J Pediatr 1970; 77:1060-2.

13. Kaner T, Sasani M, Oktenoglu T, Cirak B, Ozer AF. Postoperative spinal epidural hematoma resulting in cauda equina syndrome: a case report and review of the literature. Cases J 2009;2:8584.

14. Lawton MT, Porter RW, Heiserman JE, Jacobowitz R, Sonntag VK, Dickman CA. Surgical management of spinal epidural hematoma: relationship between surgical timing and neurological outcome. J Neurosurg 1995;83:1-7.

15. Vandermeulen EP, Van Aken H, Vermylen J. Anticoagulants and spinal-epidural anesthesia. Anesth Analg 1994;79:1165-77. 